\title{
Comparison of dynamic postural stability scores between athletes with and without chronic ankle instability during lateral jump landing
}

\author{
Zeinab Shiravi \\ Azadeh Shadmehr \\ Saeed Talebian Moghadam \\ Behrouz Attarbashi Moghadam
}

School of Rehabilitation, Tehran University of Medical Sciences, Tehran, Iran

Corresponding author:

Azadeh Shadmehr

School of Rehabilitation,

Tehran University of Medical Sciences

Piche Shemiran, Enghelab Street

1981983857 Tehran, Iran

E-mail: shadmehr@tums.ac.ir

\section{Summary}

Backgrounds: Many ankle injuries occur while participating in sports that require jumping and landing such as basketball, volleyball and soccer. Most recent studies have investigated dynamic postural stability of patients with chronic ankle instability after landing from a forward jump. The present study aimed to investigate the dynamic postural stability of the athletes who suffer from chronic ankle sprain while landing from a lateral jump.

Methods: Twelve athletes with self-reported unilateral chronic ankle instability (4 females and 8 males) and 12 matched controls ( 3 females and 9 males) voluntarily participated in the study. Dynamic postural stability index and its directional indices were measured while performing lateral jump landing test.

Results: No differences were found between athletes with and without chronic ankle instability during our landing protocol by means of the dynamic postural stability index and its directional indices. Findings showed that in each group, medial/lateral stability index is significantly higher than anterior/posterior and vertical stability indexes.

Conclusion: Findings showed that dynamic postural stability was not significantly different between the two groups. Future studies should examine chronic ankle instability patients with more severe disabilities and expose them to more chal- lenging dynamic balance conditions to further explore postural stability.

Level of evidence: Illa.

KEY WORDS: chronic ankle instability, dynamic postural stability, ground reaction forces, lateral jump landing.

\section{Introduction}

Ankle sprains are one of the most common injuries incurred by athletes ${ }^{1}$. Many ankle injuries occur while participating in sports that require jumping and landing such as basketball, volleyball and soccer ${ }^{2}$. Ankle sprains account for $1 / 6$ of all lost time from participation in sport competitions ${ }^{3}$. People who experience an ankle sprain are at risk of developing recurrent sprains. The rate of recurrence has been estimated as high as $80 \%$ among athletes ${ }^{4}$. This subjective, repeated episodes of giving way after an initial ankle sprain and deficits in neuromuscular control and mechanical stability is defined as chronic ankle instability $(\mathrm{CAI})^{5,6}$.

Dynamic postural stability is defined as an individual's ability to maintain balance while transitioning from a dynamic to a static position ${ }^{7}$. Both static and dynamic postural stability are the result of complex coordination of central processing from visual, vestibular, and somatosensory pathways, as well as the resultant efferent response ${ }^{8}$. However, postural stability, which is assessed during periods of quiet stance, may fail to reveal postural stability deficiencies because of the comparative ease of the testing procedure $^{9}$. As a result, the need to conduct more functional and dynamic testing that most closely mimics athletic performances has been emerged.

Dynamic postural control in CAI patients has been quantified through measures of lower extremity reaching distance using the Star Excursion Balance Test ${ }^{10-12}$, kinematic and kinetic assessments of jump landing 13,14 , and the ability to create stability after landing from a jump ${ }^{15-19}$

During the lateral hop stabilization task, the center of mass (COM) will oscillate primarily in the frontal plane ${ }^{20}$. Wikstrom et al. ${ }^{20}$ investigated the effects of jump direction on dynamic postural stability during landing in healthy population. They found that medial/lateral and vertical dynamic postural stability were significantly affected by the direction of the jump. More specifically, lateral and diagonal jump-landings produce increased medial/lateral stability index (ML- 
SI) scores and forward jump-landings produce increased vertical stability index (VSI) scores.

Based on the previous studies, in the lateral jump landing, the center of mass of healthy people oscillates primarily in the frontal plane, and since the lateral ankle sprain occurs most in the frontal plane ${ }^{20}$, the investigation of postural stability of this group of patients using lateral jump landing may provide valuable information on the mechanism of injury. Therefore, sole use of forward jump-landing protocols in the past, probably masked the important aspects of neuromuscular control and dynamic postural stability mechanisms which may represent the key to improve the patient treatment programs.

The previous studies on dynamic postural stability index (DPSI) measurement reported the differences between healthy people and the patients suffering from ankle instability21, 22; all the aforementioned studies, however, used forward jump landing proto$\mathrm{col}$, although there is no empirical evidence to support its use. Examining the differences in dynamic postural stability in lateral direction of jump landing, as lateral ankle sprain occurs most in frontal plane, could reveal previously overlooked information regarding neuromuscular control and may allow clinicians and researchers to develop better injury prevention strategies in CAI population. The present study, thus, aimed to investigate the dynamic postural stability of the athletes who suffer from chronic ankle sprain while landing from a lateral jump.

\section{Methods}

\section{Subjects}

Twelve control subjects without CAI ( 3 females and 9 males) and 12 subjects with CAI (4 females and 8 males) voluntarily participated in the study. They were matched according to age, body mass, height, type and duration of physical activity, and lower-limb dominance (Tab. I). Participants were physical education students of Tehran University exercising regularly three times a week for at least $2 \mathrm{~h}$ each time. All subjects were college basketball, volleyball or football players. Limb dominance was determined by the preferred limb used to kick a ball in each group: 10 subjects were tested on their nondominant limb and 2 subjects were tested on dominant limb.

Subjects with CAI from 18 to 30 years old included if they had at least one significant unilateral inversion ankle sprain with pain, swelling, and loss of function within the last one year, followed by repeated injuries or any sign of ankle instability or "giving-way". Subjects in the CAI group had a range of 1 to 3 ankle sprains within the previous 12 months and did not undergo rehabilitation. The information was gathered by a trained physical therapist using self-report questionnaire and clinical examination. Individuals in the control group were excluded if they reported a history of ankle sprain or perception of giving-way in the ankle. Subjects in both groups were excluded if they had cardiovascular disease, vestibular or respiratory disorder, auditory or cognitive deficit, diabetes, or recent lower-limb or low back pathology, or were using any medicine that could affect their balance.

All participants signed an informed consent form which had been approved by the Ethics Committee of Tehran University of Medical Sciences before commencing the study. The study meets the ethical standards of the Journal23.

\section{Experimental design}

All data were collected by a strain gauge Bertec 9090-10 force platform and a Bertec AM-6701 amplifier (Bertec Corp., Columbus, $\mathrm{OH}$ ). COP data were collected with a sampling frequency of $500 \mathrm{~Hz}$.

Force plate data were initially recorded during a relatively simple dynamic choice reaction task (CRT). All participants performed warm up exercises before the initiation of testing. Before the main trial, they were asked to perform lateral jump landing task on either the dominant or the nondominant limb to their maximum length three times, and then $75 \%$ of the maximum jump distance was used for the test. Subjects were allowed as many practice trials as needed to feel comfortable with the jump protocol and a 2minute rest period before completing the testing protocol to prevent fatigue.

Participants started the test by standing barefoot on the force plate, at a determined distance $(75 \%$ maximum jump) away from the center of the force plate. $A$ box, set according to the height of individuals, located $1 \mathrm{~m}$ away in front of their eyes. Red and green lamps which turn on randomly by the tester were sat in the box. Participants were required to jump as quickly as possible from this position to the force plate if the green lamp turn on and keep their balance for 5 seconds after landing; conversely, participants were instructed to stand still when the red lamp turned on. Each subject was asked to land on the test leg, stabilize as quick as possible, and balance for 5 seconds with hands on the hips looking straight ahead. Jumping was repeated three times and the mean values were used for final analysis. In sum, each participant was randomly exposed to 12 experimental conditions.

\section{Data analysis}

Anterior/posterior (AP), medial/lateral (ML), vertical (V) and dynamic postural (DP) stability indices (SI) quantified dynamic postural control ${ }^{19}$. The MLSI and APSI assess the GRF fluctuations from 0 along the frontal and sagittal axes of the force plate, respectively. The VSI assesses the fluctuation from the subject's body weight to standardize the vertical GRF along the vertical axis of the force plate. This is done to normalize the vertical scores among individuals with different body weights (mass). The dynamic postural stability index (DPSI) is a composite of the ML$\mathrm{SI}, \mathrm{APSI}$, and VSI and is sensitive to changes in all 3 directions ${ }^{19}$. 
Comparison of dynamic postural stability scores between athletes with and without chronic ankle instability during lateral jump landing

\section{Statistical analysis}

All data were analyzed using SPSS software version 20. A one-sample Kolmogorov Smirnov test was performed to determine normal distribution of all variables ( $p>0.05)$. Reliability analysis for all data was performed by ICC in each group consisting of all subjects. The mean of the three trials of data gathering was used for statistical analysis. Comparisons between the 2 groups were made with independent $t$ tests for age, weight, height and body mass index. The mean of the 3 trials for the measurements of stability indices was used for statistical analysis. Separate 2-by-2 [group (CAl and control) by limb (dominant and nondominant] mixed model ANOVAs were used for each postural stability index. All dependent variables were analyzed using multivariate ANOVA. An alpha level of 0.05 was used for all statistical tests with a Bonferroni adjustment.

\section{Results}

There were no significant differences in age $(p=0.32)$, height $(p=0.94)$, weight $(p=0.56)$ and body mass in$\operatorname{dex}(p=0.43)$ between the 2 groups (Tab. I). Values of Kolmogorov-Smirnov showed that the distribution of all variables in both groups were normal. The results of reliability analysis (ICC) are available on Table II. ICC values were interpreted according to Munro's classification of reliability: 0.26 to 0.49 reflects a low correlation, 0.50 to 0.69 reflects a moderate correlation, 0.70 to 0.89 reflects a high correlation and 0.90 to1.00 indicates a very high correlation (Domholdt, 2005). Except for APSI of dominant leg of healthy group, for which a moderate correlation was found, all parameters had high or very high correlations.

Table III shows the mean and SD of dynamic stability measures for both groups. A summary of ANOVA results for the four measures of dynamic stability is shown in Table IV. Findings showed that the main effects of group and limb and interactions of group by limb were not significant differences for any of the dependent variables (Tab. IV).

Findings showed in each group, MLSI is significantly higher than APSI and VSI, also APSI is significantly higher than VSI (MLSI>APSI>VSI) (Fig. 1).

\section{Discussion}

This is the first study that measured dynamic postural stability scores of athletes with chronic ankle instability after landing from lateral jumping. In spite of the small sample size, our pilot study demonstrated a high to very high level of reliability for the measurements of stability indexes in lateral jumping. Wikstrom 2005 found very high reliability of DPSI measures (ICC=0.96) for forward jumping protocol.

No differences were found between athletes with and

Table I. Demographic data of athletes with chronic ankle instability $(n=12)$ and healthy athletes $(n=12)$.

\begin{tabular}{cccc}
\hline & $\begin{array}{c}\text { CAI } \\
\text { Mean (SD) }\end{array}$ & $\begin{array}{c}\text { Healthy } \\
\text { Mean (SD) }\end{array}$ & P value \\
\hline Age (year) & $22.50(3.26)$ & $24.08(4.37)$ & 0.32 \\
Height $(\mathrm{cm})$ & $174.5(9.31)$ & $174.8(9.68)$ & 0.94 \\
Weight $(\mathrm{kg})$ & $69.66(11.78)$ & $72.75(13.82)$ & 0.56 \\
Body mass index $\left(\mathrm{kg} / \mathrm{cm}^{2}\right)$ & $22.72(2.47)$ & $23.62(3.03)$ & 0.43 \\
\hline
\end{tabular}

Abbreviations: CAI, Chronic Ankle Instability.

Table II. Reliability analysis (average ICC) of Dynamic Postural Stability Index, and directional components in athletes with chronic ankle instability $(n=12)$ and healthy athletes $(n=12)$.

\begin{tabular}{ccccccccc}
\hline & \multicolumn{3}{c}{ Healthy } & & \multicolumn{3}{c}{ CAI } \\
& \multicolumn{2}{c}{ ICC (95\% $\mathbf{~ C l )}$} & \multicolumn{2}{c}{ Sig. (2- tailed) } & \multicolumn{2}{c}{ ICC (95\% Cl) } & \multicolumn{2}{c}{ Sig. (2- tailed) } \\
\hline Variables & Nondominant & Dominant & Nondominant & Dominant & Injured & Noninjured & Injured & Noninjured \\
\hline APSI & 0.95 & 0.69 & 0.000 & 0.009 & 0.87 & 0.95 & 0.000 & 0.000 \\
MLSI & 0.99 & 0.97 & 0.000 & 0.000 & 0.99 & 0.98 & 0.000 & 0.000 \\
VSI & 0.97 & 0.70 & 0.000 & 0.007 & 0.95 & 0.96 & 0.000 & 0.000 \\
DPSI & 0.97 & 0.87 & 0.000 & 0.000 & 0.94 & 0.96 & 0.000 & 0.000 \\
\hline
\end{tabular}

Abbreviations: CAI, Chronic Ankle Instability; APSI, Anterior/Posterior Stability Index; MLSI, Medial/Lateral Stability Index VSI, Vertical Stability Index; DSI, Dynamic Postural Stability Index. 
Table III. Mean and standard deviation of Dynamic Postural Stability Index, and directional components in athletes with chronic ankle instability (CAI) $(n=12)$ and healthy athletes $(n=12)$.

\begin{tabular}{ccccc}
\hline \multirow{2}{*}{ Variables } & \multicolumn{2}{c}{ Healthy } & \multicolumn{2}{c}{ CAI } \\
\cline { 2 - 5 } & Dominant & Nondominant & Noninjured & Injured \\
\hline APSI & $0.08(0.07)$ & $0.08(0.08)$ & $0.11(0.11)$ & $0.11(0.10)$ \\
MLSI & $0.31(0.07)$ & $0.31(0.06)$ & $0.30(0.04)$ & $0.30(0.04)$ \\
VSI & $0.02(0.02)$ & $0.02(0.03)$ & $0.03(0.04)$ & $0.03(0.04)$ \\
DPSI & $0.33(0.08)$ & $0.34(0.07)$ & $0.34(0.07)$ & $0.34(0.07)$ \\
\hline
\end{tabular}

Abbreviations: CAI, Chronic Ankle Instability; APSI, Anterior/Posterior Stability Index; MLSI, Medial/Lateral Stability Index VSI, Vertical Stability Index; DSI, Dynamic Postural Stability Index.

Table IV. Summary of analysis of variance for measures of dynamic stability in lateral jump landing.

\begin{tabular}{|c|c|c|c|c|c|c|c|c|}
\hline \multirow{2}{*}{$\begin{array}{c}\text { Independent } \\
\text { variable }\end{array}$} & \multicolumn{2}{|c|}{ APSI } & \multicolumn{2}{|c|}{ MLSI } & \multicolumn{2}{|c|}{ VSI } & \multicolumn{2}{|c|}{ DPSI } \\
\hline & F ratio & $P$ value & F ratio & $P$ value & F ratio & $P$ value & F ratio & $P$ value \\
\hline Group & 0.532 & 0.470 & 0.297 & 0.589 & 0.723 & 0.400 & 0.070 & 0.792 \\
\hline Limb & 0.001 & 0.981 & 0.086 & 0.770 & 0.024 & 0.878 & $0 / 029$ & 0.865 \\
\hline $\begin{array}{l}\text { Interaction } \\
\text { Group Limb }\end{array}$ & 0.039 & 0.844 & 0.044 & 0.834 & 0.019 & 0.890 & 0.079 & 0.780 \\
\hline
\end{tabular}

Abbreviations: CAI, Chronic Ankle Instability; APSI, Anterior/Posterior Stability Index; MLSI, Medial/Lateral Stability Index VSI, Vertical Stability Index; DSI, Dynamic Postural Stability Index.

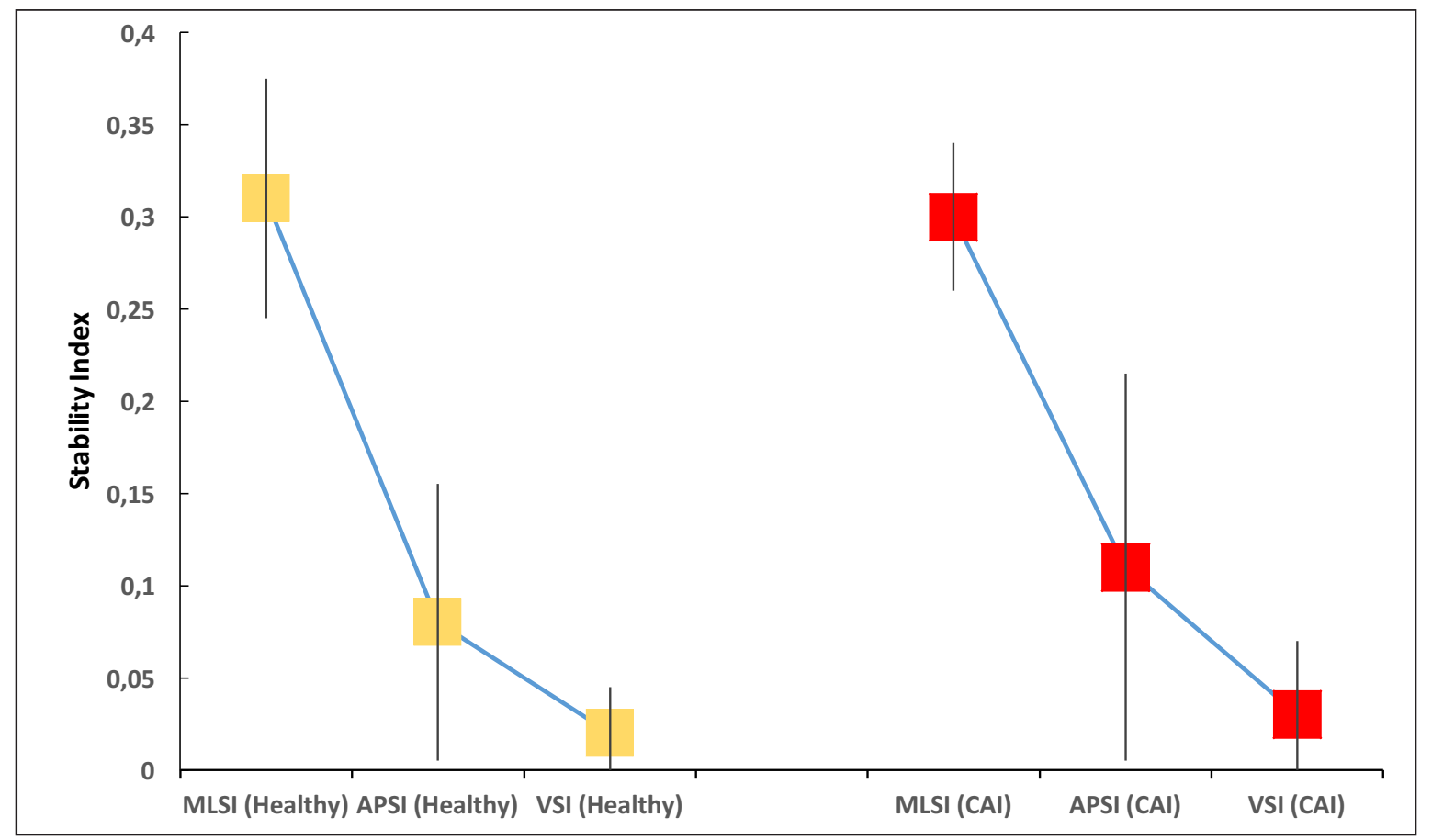

Figure 1. Interaction plots showing postural stability indices as a function of group and directional indices of dynamic postural stability. MLSI>APSI>VSI in healthy and CAI groups.

Abbreviations: CAI, Chronic Ankle Instability; APSI, Anterior/Posterior Stability Index; MLSI, Medial/Lateral Stability Index; VSI, Vertical Stability. 
without chronic ankle instability during our landing protocol by means of the DPSI and its directional indices. These findings are inconsistent with previous studies $21,22,24$.

Previous studies examined dynamic postural stability during forward jumping. It is possible that APSI and VSI scores were not affected because of the mediolateral orientation of the landing task. Wikstrom et al. ${ }^{20}$ found a statistically significant increase in MLSI scores as the nature of the jump-landing protocol became more lateral. However the MLSI score was not different between two groups. It could be due to the fact that our participants were athletes who involved in sport activities despite their ankle injury. Therefore, selecting more impaired CAI patients may be helpful to better understanding dynamic postural stability in lateral jumping.

On the other hand, it is possible that no statistical differences were observed between groups due to altered balance strategies. According to Pintsaar et al. ${ }^{25}$ individuals suffering ankle instability apply a hip strategy technique to compensate for the lack of stability in the ankle during a single leg balance task. It seems that because of lateral orientation of the landing protocol, abductor and adductor muscles of the hip and trunk were able to successfully compensate for the decreased neuromuscular control of the ankle. Therefore, it is possible that our patients used a trunk strategy in the frontal plane as a compensatory mechanism to maintain balance after the jump. However, more studies are required investigating EMG and joint kinematics to prove this hypothesis.

The current investigation noted a statistically significant increase in MLSI compared to APSI and VSI scores as the nature of the jump-landing protocol was lateral (i.e. MLSI scores > APSI scores > VSI scores) in both patients and healthy athletes. These results are in line with Wikstrom et al. $^{20}$, that indicated dynamic postural stability is affected by the direction of the jump landing used. They found that in a healthy population, jump direction will statistically affect dynamic postural stability in the frontal and vertical planes. During lateral jump landing task, the COM oscillates primarily in the frontal plane, explaining the results of Wikstrom ${ }^{20}$, as well as the MLSI findings in the current investigation. Despite the same results in patients and control groups, these findings may have significant clinical outcomes, considering the extremely high incidence of lateral ankle sprains and that lateral ankle sprains occur in the frontal plane.

Differences in study design (i.e. providing visual stimulus and using lateral jumping protocol), sample size, and target population could be the sources of different findings in this study.

A potential limitation of the present study is the lack of information about the level of disability experienced by subjects with CAI. This means that comparison of our results with other studies in the area is difficult due to the wide variability in subjects presenting with a diagnosis of CAI.

In conclusion, these findings showed that dynamic postural stability was not significantly different between the two groups. Future studies should examine CAl patients with more severe disabilities and should expose them to more challenging dynamic balance conditions to further explore postural stability.

\section{Conflict of interest}

The Author has no financial or personal relationships with other people or organizations that could inappropriately influence their work.

\section{References}

1. Hoch MC, McKeon PO. Peroneal reaction time after ankle sprain: a systematic review and meta-analysis. Med Sci Sports Exerc. 2014;46(3):546-556.

2. Wikstrom EA, Tillman MD, Borsa PA. Detection of dynamic stability deficits in subjects with functional ankle instability. Med Sci Sports Exerc. 2005;37(2):169-175.

3. Wright I, Neptune R, van den Bogert AJ, Nigg B. The influence of foot positioning on ankle sprains. Journal of biomechanics. 2000;33(5):513-519.

4. Rahnama L, Salavati M, Akhbari B, Mazaheri M. Attentional demands and postural control in athletes with and without functional ankle instability. Journal of orthopaedic \& sports physical therapy. 2010;40(3):180-187.

5. Brown CN, Padua DA, Marshall SW, Guskiewicz KM. Hip kinematics during a stop-jump task in patients with chronic ankle instability. Journal of athletic training. 2011;46(5):461-467.

6. Hertel J. Functional anatomy, pathomechanics, and pathophysiology of lateral ankle instability. Journal of athletic training. 2002;37(4):364.

7. Goldie PA, Bach T, Evans O. Force platform measures for evaluating postural control: reliability and validity. Archives of physical medicine and rehabilitation. 1989;70(7):510-517.

8. Palmieri RM, Ingersoll CD, Cordova ML, Kinzey SJ, Stone MB, Krause BA. The effect of a simulated knee joint effusion on postural control in healthy subjects. Archives of physical medicine and rehabilitation. 2003;84(7):1076-1079.

9. Riemann BL, Caggiano NA, Lephart SM. Examination of a clinical method of assessing postural control during a functional performance task. JSR. 2010;8(3).

10. Gribble P, HertelJ, Denegar C. Chronic ankle instability and fatigue create proximal joint alterations during performance of the Star Excursion Balance Test. International journal of sports medicine. 2007;28(3):236-242.

11. Gribble PA, Hertel J, Denegar CR, Buckley WE. The effects of fatigue and chronic ankle instability on dynamic postural control. Journal of Athletic Training. 2004;39(4):321.

12. Olmsted LC, Carcia CR, Hertel J, Shultz SJ. Efficacy of the Star Excursion Balance Tests in detecting reach deficits in subjects with chronic ankle instability. Journal of athletic training. 2002;37(4):501.

13. Caulfield B, Garrett M. Functional instability of the ankle: differences in patterns of ankle and knee movement prior to and post landing in a single leg jump. International journal of sports medicine. 2002;23(1):64-68.

14. Caulfield B, Garrett M. Changes in ground reaction force during jump landing in subjects with functional instability of the ankle joint. Clinical biomechanics. 2004;19(6):617-621.

15. Brown C, Ross S, Mynark R, Guskiewicz K. Assessing functional ankle instability with joint position sense, time to stabilization, and electromyography. Journal of Sport Rehabilita- 
tion. 2004:122-134.

16. Brown CN, Mynark R. Balance deficits in recreational athletes with chronic ankle instability. Journal of athletic training. 2007; 42(3):367-374.

17. Ross SE, Guskiewicz KM. Examination of static and dynamic postural stability in individuals with functionally stable and unstable ankles. Clinical Journal of Sport Medicine. 2004;14 (6):332-338.

18. Ross SE, Guskiewicz KM, Yu B. Single-leg jump-landing stabilization times in subjects with functionally unstable ankles. Journal of athletic training. 2005;40(4):298.

19. Wikstrom EA, Tillman MD, Smith AN, Borsa PA. A new forceplate technology measure of dynamic postural stability: the dynamic postural stability index. Journal of Athletic Training. 2005;40(4):305.

20. Wikstrom EA, Tillman MD, Schenker SM, Borsa PA. Jumplanding direction influences dynamic postural stability scores. Journal of Science and Medicine in Sport. 2008;11(2):106111.

21. Wikstrom EA, Tillman M, Chmielewski T, Cauraugh J, Naugle
K, Borsa P. Dynamic postural control but not mechanical stability differs among those with and without chronic ankle instability. Scandinavian journal of medicine \& science in sports. 2010;20(1):e137-e44.

22. Wikstrom EA, Tillman MD, Chmielewski TL, Cauraugh JH, Borsa PA. Dynamic postural stability deficits in subjects with self-reported ankle instability. Medicine and science in sports and exercise. 2007;39(3):397.

23. Padulo J, Oliva F, Frizziero A, Maffulli N. Muscles, Ligaments and Tendons Journal - Basic principles and recommendations in clinical and field science research: 2016 update. MLTJ. 2016;6(1):1-5.

24. De Ridder R, Willems T, Vanrenterghem J, Roosen P. Effect of tape on dynamic postural stability in subjects with chronic ankle instability. International journal of sports medicine. 2015; 36(04):321-326.

25. Pinstaar A, Brynhildsen J, Tropp H. Postural corrections after standardized perturbations of single leg stance: effect of training and orthotic devices in patients with ankle instability. $\mathrm{Br} \mathrm{J}$ Sports Med. 1996;30:151-155. 